\title{
Planning pesticides usage for herbal and animal pests based on intelligent classification system with image processing and neural networks
}

\author{
Kamil Dimililer ${ }^{1, *}$, Yoney Kirsal Ever ${ }^{2}$ \\ ${ }^{1}$ Electric and Electronic Engineering Department, Near East University, North Cyprus, Mersin 10 Turkey \\ ${ }^{2}$ Software Engineering Department, Near East University, North Cyprus, Mersin 10 Turkey
}

\begin{abstract}
Pests are divided into two as herbal and animal pests in agriculture, and detection and use of minimum pesticides are quite challenging task. Last three decades, researchers have been improving their studies on these manners. Therefore, effective, efficient, and as well as intelligent systems are designed and modelled. In this paper, an intelligent classification system is designed for detecting pests as herbal or animal to use of proper pesticides accordingly. The designed system suggests two main stages. Firstly, images are processed using different image processing techniques that images have specific distinguishing geometric patterns. The second stage is neural network phase for classification. A backpropagation neural network is used for training and testing with processed images. System is tested, and experiment results show efficiency and effective classification rate. Autonomy and time efficiency within the pesticide usage are also discussed.
\end{abstract}

\section{Introduction}

Last three decades, people have been concerned about health and safety of the food consuming. Since there are several hundreds of pest species in paddy fields, various types of pesticides are applied but these health and safety awareness make the agricultural staff to minimize the use of pesticides in necessary amount. In order to decide the required pesticide, visual inspection and comparison of insects is commonly used method around the world. This approach is highly challenging and requires considerable training and experience with insects. The experimental methods are time-consuming, affordable, and not suitable for routine use [27].

Recently, an increment in the usage of image processing techniques in various applications as preprocessing stage are seen [29-31]. Image processing techniques have been applied in different ways to identify crops, plants, leaves as well as diseases in agriculture paddy fields [1]. According to their research, an image segmentation technique is used for detection of agricultural products. In [24] paper, a short survey for potential future techniques for image processing in different agricultural areas were highlighted to guide researchers and farmers. [23] did a similar study to increase the productivity while reducing the diseases. In another image processing research, a software was developed to decide different vegetation indexes and the 3-dimensional feature of crops by [20].
Artificial Neural Networks is widely used in the classification of different objects [30, 31]. In order to classify pests, biometric based features are adopted for automatically identifying and classifying the images of insects. The primary advantage of this approach is that it is invariant to changes in pose and scale. Furthermore, to achieve a high classification accuracy, a backpropagation neural classifier is required for detection.

The main aim of this research is to classify different types of pests based on their geometric features that are extracted using edge detection. The classification is performed using a backpropagation neural network due to its simplicity and efficiency in such applications [10]. Different image processing techniques are applied to images in order to highlight the edges and prepare them for the back propagation neural networks. The images are prepared for simulation and testing purposes [7]. Therefore, three backpropagation neural networks are used. Two types of edge detection methods have been applied to the available image set after applying the median filtering in order to extract the features of the insects. The prepared image set will be prepared for the input layer of the Back Propagation Neural Networks, BPNN. The simulation results show differences in the classification rates and effectiveness of the suggested networks.

The experiment demonstrates a classification success rate of $84 \%$ for the first network, $90 \%$ for the second network and $92 \%$ for the third network. The 
method developed in this paper offers potential for the classification of pests.

\section{Related Works}

In literature, there are different types of neural network algorithms used for categorization or classification of various images [15]. In this paper, the proposed system is divided into two categories; image processing stage and neural networks stage. The images are classified as herbal and animal pests such as Agrotis Ipsilon, Gryllotalpa, carponya, tetrancyhidae, Mamestra, Lertinotarsa, Siphonaptera, ixodida, Siphonaptera, Cimicidae, Phlebotomus, Aphidoidea. The image processing phase consists of grayscale conversion, filtering, Sobel and Canny edge detection. These techniques are used to highlight the geometric shapes of the pests taking into account the distinguished features. At the end of this phase, the images are ready to be fed into the proposed neural network phase.

\subsection{Dataset}

Due to the unavailability of a single source of insect images, the images were collected from different websites for insect classification. Resultantly, the total number of images was 120 , out of which 10 were Agrotis, 10 were Gryllotalpa, 10 were Carpomya, 10 were Tetranychidae, 10 were Mamestra, 10 were Lepinotarsa, 10 were Siphonaptera, 10 were ixodida, 10 were Siphonaptera, 10 were Cimicidae, 10 were Phlebotomus. 10 were Aphidoidea. Figure 1. Shows the sample database images.

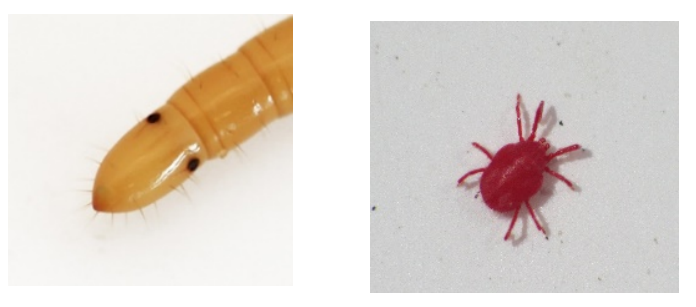

(a) Herbal Pests

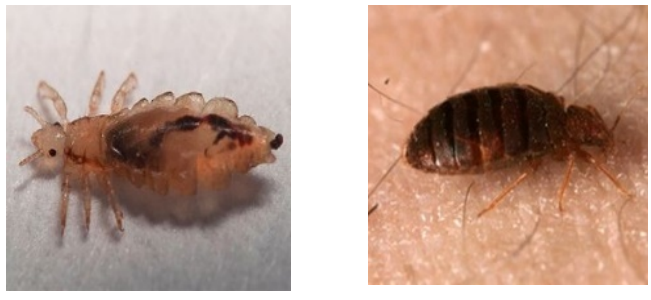

(b) Animal Pests

Fig. 1. Sample images from the database.

\section{Proposed Systems}

This section focusses on utilities and parameters of the proposed system. Two phases, image processing and neural network phases are designed respectively.

\subsection{Image Processing Phase}

A bunch of image processing methods is applied to the pest database for acceptable amount of identification.

\subsubsection{Formatting the title}

In order to minimize the CPU Time, the original RGB images are converted to grayscale. Using the luminosity method, grayscale images are more precise [3]. Figure 2 shows the conversion of a colour image into a grayscale image.

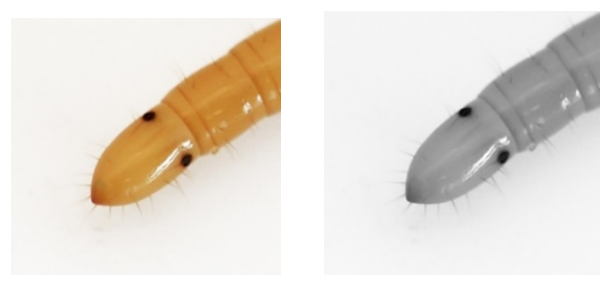

(a) Herbal Pests

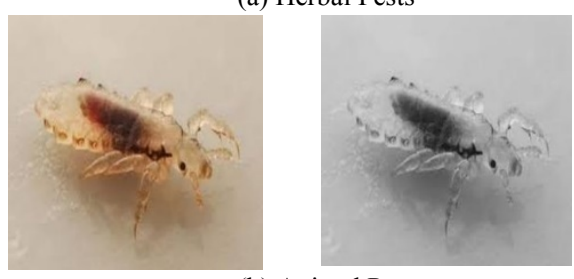

(b) Animal Pests

Fig. 2. RGB to Grayscale conversion.

\subsubsection{Image smoothing with filtering}

Smoothing is one of the image processing techniques used for reducing the details within an image in order to create a clearer image [3]. The most common type of linear filter is the median filter which is used to reduce impulsive noise [6]. The median filter goes through each element of the image and replaces each pixel with the median of its neighboring pixels that are located in a square neighborhood (kernel) around the evaluated pixel [25]. Figure 3 represents the application of image filtering to the pest insects.
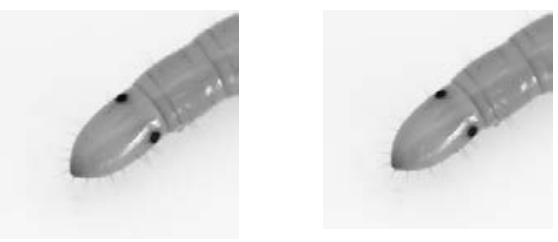

(a) Herbal Pests
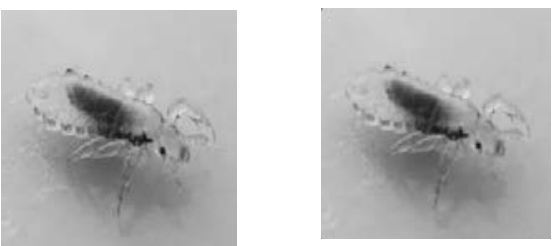

(b) Animal Pests

Fig. 3. Filtering applied images. 


\subsubsection{Thresholding}

Image thresholding separates a particular region of image into two regions. First region is the region for the foreground, where second one is the background region for the unneeded objects. In proposed system, the input data is a grayscale image, where the output is a binary image that [0] (black pixels) represents background and [1] (white pixels) represents foreground. Intensity threshold is set by analyzing the histogram of the image.

This represents the intensity distribution of that particular image [10]. Figure 4 represents the application of thresholding to the filtered grayscale image.

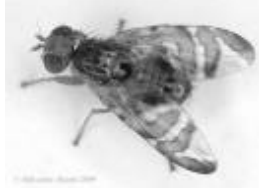

(a) Herbal Pests
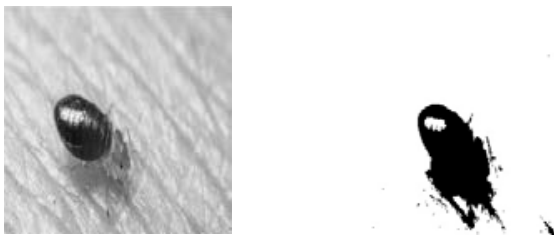

(b) Animal Pests

Fig. 4. Threshold applied images.

\subsubsection{Edge detection based segmentation}

Segmentation is defined as grouping of required regions of the image into foreground and background regions of interest and removed data respectively [8].

Edge detection is identified as sharp intensity contrasts in an image. In this proposed system, edges are detected using Canny and Sobel operators. Canny edge detection technique provides good noise immunity and detects the through edges while minimizing errors. On the other hand, Sobel edge detection technique uses 3by-3 kernels which calculates approximation of the horizontal and vertical derivatives [2,14]. Figure 5 shows the segmentation applied images using canny and sobel operators.

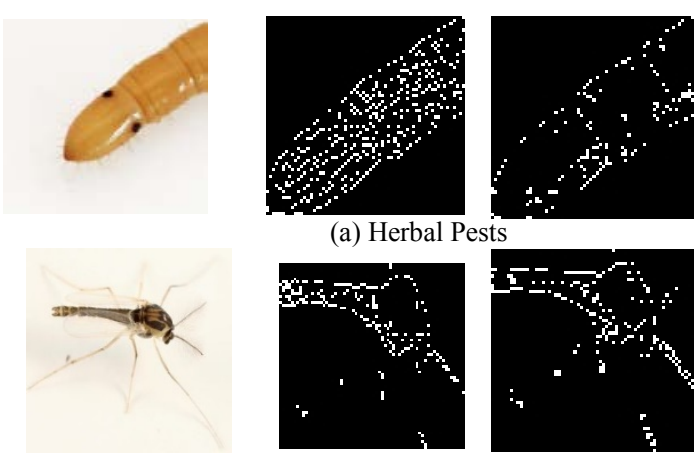

(b) Animal Pests

Fig. 5. Segmentation applied images.

\subsection{Neural Networks Phase}

In this phase, the images of the different pests were classified into two classes using a supervised backpropagation neural classifier. One database was used for training and testing the different networks. However, three learning schemes were used $[4,5]$.

The proposed system consists of a database of 60 images for herbal pests and 60 images for animal pests where 70 images are used for training purpose whereas 50 images were used for testing. There are 3 BPNN Learning schemes used to test the efficiency of the suggested application. 65536 pixels represents the $256 \times 256$ grayscale image that is used as the input data.

Figure 6 represents the 3 topologies of the suggested neural network phases where Figure 6(a) shows the original grayscale images as inputs, Figure 6(b) shows the Canny edge detection applied images as inputs, and Figure 6(c) shows the Sobel edge detection applied images as inputs.

Table 1. BPNN Training final parameters.

\begin{tabular}{|c|c|c|c|}
\hline & BPNN1 & BPNN2 & BPNN3 \\
\hline & (Fig.6(a)) & (Fig.6(b)) & (Fig.6(c)) \\
\hline $\begin{array}{c}\text { Input layer } \\
\text { nodes }\end{array}$ & 65536 & 65536 & 65536 \\
\hline $\begin{array}{c}\text { Output layer } \\
\text { nodes }\end{array}$ & 2 & 2 & 2 \\
\hline $\begin{array}{c}\text { Learning } \\
\text { rate }\end{array}$ & 0.004 & 0.004 & 0.003 \\
\hline $\begin{array}{c}\text { Momentum } \\
\text { rate }\end{array}$ & 0.3 & 0.5 & 0.5 \\
\hline $\begin{array}{c}\text { Minimum } \\
\text { error }\end{array}$ & 0.002 & 0.002 & 0.002 \\
\hline
\end{tabular}

\section{Results and Discussions}

These results were obtained using a configuration of 2012R2 64 bits OS Virtual machine that has a 1 Gbits connection speed to a super computer that has IBM Blade HS21 XM Intel Xeon $2.33 \mathrm{GHz}$ CPU with 1280 core units. Through this application, the robustness, flexibility and speed of this pest detection system have been demonstrated. Pests identification results using the training image set yielded $100 \%$ recognition as would be expected in BPNN.

Figure 6 shows the topologies of the suggested BPNN Systems. Due to the implementation simplicity, and the availability of sufficient "input - target" database for training, the use of a back propagation neural network which is a supervised learner, has been preferred.

Training and testing (generalization) are comprised for these phases. The available brain image database is organized as follows: 

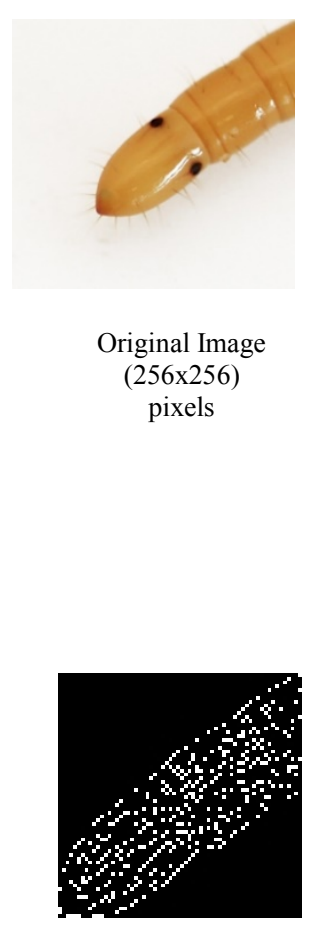

Canny Edge Detection Applied Image (256x256) pixels

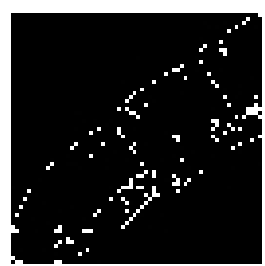

Sobel Edge Detection Applied Image (256x256)

pixels
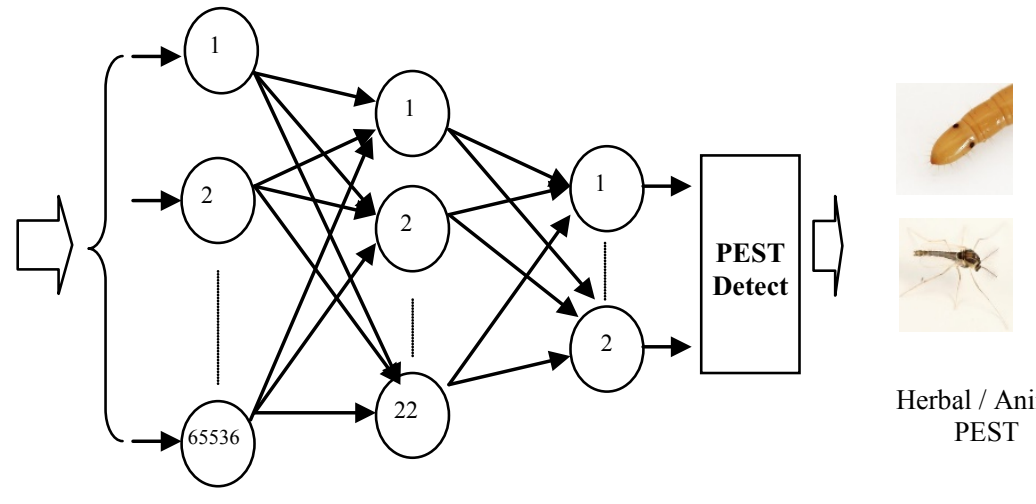

Herbal / Animal PEST

(a)
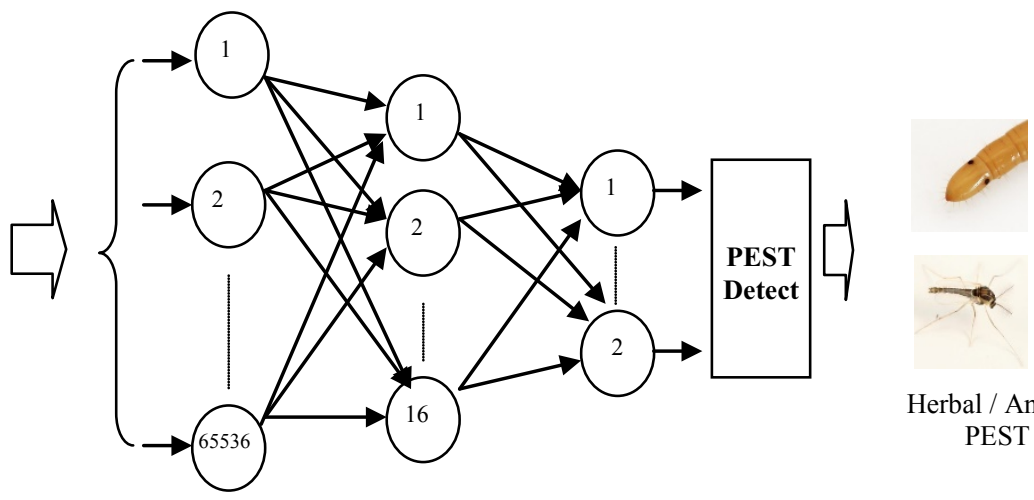

Herbal / Animal PEST

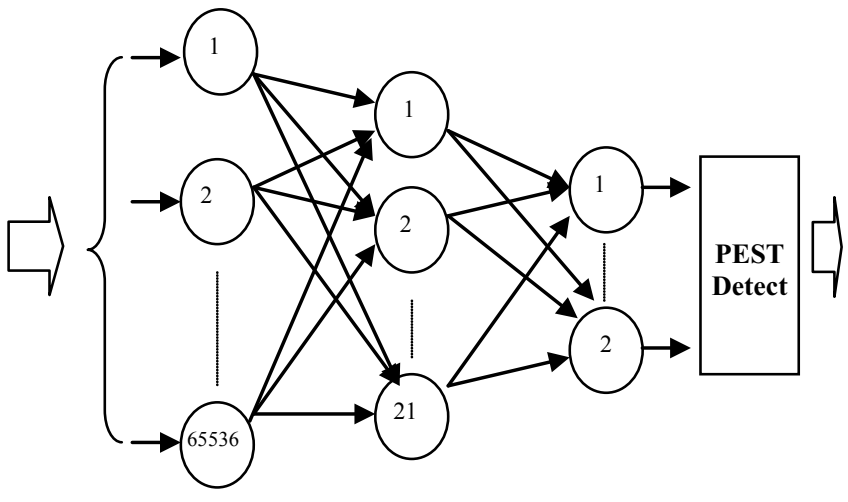

(c)
Fig.6. BPNN Architectures.

1. Training image set: 70 images

2. Testing image set: 50 images

During the learning phase, initial random weights of values between -0.3 and 0.3 were used. In order to achieve the required minimum error value and meaningful learning, the learning rate and the momentum rate were adjusted during various experiments.

In BPNN1, the identification of the images is yielded $84 \%$ where, 42 images out of the available 50 pest images correctly yielded, in BPNN2, the identification of the images is yielded $90 \%$ where, 45 images out of the available 50 pest images correctly yielded whereas in BPNN3, the identification of the images is yielded $92 \%$ where, 46 images out of the available 50 pest images correctly yielded.

As it can be seen from the results of the iterations, results were successful and encouraging. BPNN3 which represents Sobel edge detection algorithm yields optimum results, after several experiments. This successful result was obtained by using only the database of images for training the neural network. 


\section{Conclusion and Future Works}

In this paper, image processing and neural network approaches for the detection of pests were developed. Image processing techniques are very useful and significant in various application fields.

The proposed system uses three back propagation neural networks, named as BPNN1, BPNN2, BPNN3. The developed BPNNs use pre-processed, original images to detect pests with and without fractures respectively.

The motivation of this work was to compare and evaluate the obtained results and also, effectiveness of a back propagation neural network in recognizing different herbal and animal pests. Comparing the identification rations as discussed in the results and discussions section, BPNN3 that is Sobel edge identification.

The developed system is a robust system since it was trained to recognize pests. The images used for training and testing the proposed system were collected from the benchmark database, which contains 120 images. The images contain different features in size and illumination conditions for each subject, which was an advantage for us to make the system more efficient and robust. Finally, it can be stated that the experimental analysis of the proposed pests detection system showed a great efficiency and an outperforming rate.

Last but not the least, the proposed BPNN algorithms results will guide architectural staff in term of detection and determination of the most efficient and least harmful pesticides.

\section{References}

1. R. Chahar and P. Soni A study of image processing in agriculture for detect the plant diseases. International Journal of Computer Science and Mobile Computing. 4:581-587, (2015).

2. L., Chao, Z., Ji-liu and H.E., Kun, Adaptive edge-detection method based on Canny algorithm. Computer Engineering and Design, 31:4036-4039, (2010).

3. J., Church, Y., Chen, and S., Rice, A Spatial median filter for noise removal in digital images, IEEE Southeast Conf., 618 - 623, (2008).

4. K. Dimililer, Backpropagation neural network implementation for medical image compression. J. Appl. Math., (2013).

5. K. Dimililer, Neural network implementation for image compression of x-rays. Electronics World. 118:26-29, (2012).

6. K. Dimililer, and A. İlhan, Effect of image enhancement on mri brain images with neural networks, (2016).
7. K., Dimililer, Y. Kirsal Ever, and H. Ratemi, Intelligent eye tumour detection system. Procedia Computer Science, 102:325-332, (2016a).

8. K. Dimililer, Y. Kirsal Ever, and B. Ugur, ILTDS: Intelligent lung tumor detection system on ct images. Intelligent Systems Technologies and Applications, 225-235, (2016b).

9. K. Dimililer, Y. Kirsal Ever and B. Ugur, Tumor Detection on CT Lung Images using Image Enhancement, The Online Journal of Science and Technology, 7, (2016c).

10. K. Dimililer and S. Zarrouk, ICSPI: Intelligent classification system of pest insects based on image processing and neural arbitration, American Society of Agricultural and Biological Engineers, 33:4, (2017).

11. Y. K. Ever, E. Gemikonakli, and K. Dimililer, Modelling Approaches of Performance Evaluation Of High QoS Of Kerberos Server With Dynamically Renewing Keys Under Pseudo Conditions, The Online Journal of Science and Technology, 7, (2016).

12. H.L, Eng and K.K, Ma, Noise adaptive softswitching median filter. IEEE Trans Image Process, 10:242-251, (2001).

13. Gonzalez and Woods, Digital Image Processing, Second Edition, Prentice Hall,108120, (2002).

14. Z., Jian-jun, and L. Jing, A surface crack edge detection algorithm based on improved Sobel operator. Journal of hefei university of technology, 34:845- 847,960, (2011).

15. A. Khashman and K. Dimililer, Medical radiographs compression using neural networks and haar wavelet, IEEE EUROCON'09, 14481453, (2009).

16. Y. Kim, Contrast enhancement using brightness preserving bi-histogram equalization, IEEE Trans. Consum. Electron. 43:1-8, (1997).

17. N., Larios, Deng, H., Zhang, W., Sarpola, M., Yuen, J., Paasch, R., Moldenke, A., Lytle, D. A., Correa, S. R., Mortensen, E. N., Shapiro, L. G., and Dietteric, T.G., Automated insect identification through concatenated histograms of local appearance features: feature vector generation and region detection for deformable objects, Machine Vision and Applications, Springer, 19:105-123, (2008).

18. T.Z. Lee, Edge detection analysis. Int. J. Comp. Sci., 5:1-25, (2012). 
19. I., Levner and H. Zhangm. Classification driven watershed segmentation, IEEE Transactions on image Processing, 16, (2007).

20. A. F. J., Lopez, Salamanca, J. M., Medina, M. J. Q., and Perez, O. E. A. Crops diagnosis using digital image processing and precision agriculture technologies., INGE CUC. 11:6371, (2015).

21. A. Mittal and, S.K., Dubey Analysis of rheumatoid arthritis through image processing. IJCSI International Journal of Computer Science. 9, (2012).

22. R.G., Mundada, and, V. V. Gohokar, Detection and classification of pests in greenhouse using image processing, IOSR Journal of Electronics and Communication Engineering. 5:57-63. Procedia Computer Science, 102:39-44, (2013).

23. J. K., Patil, and R. Kumar. Advances in image processing for detection of plant diseases, Journal of Advanced Bio information Applications and Research, 135-141, (2011).

24. L. P. Saxena and L. J. Armstrong, A survey of image processing techniques for agriculture. Proceedings of AFITA 2014, 406-418, (2014).

25. L. Shapiro and G, Stockman, Image Segmentation. (1st ed.), Prentice Hall, Washington, pp. 200-280, (2000)..

26. The nature pictures, available at: [http://www.naturespic.com/NewZealand/result keyword.asp?imagekeywords $=$ Insects $\% 2$ Fspid ers\&offset $=60]$.

27. L. G., Thiago, Souza, E.S., Mapa, K.S., and David M. Application of complex networks for automatic classification of damaging agents in soya bean leaflets. Proceeding of the 18th IEEE International Conference, 1065 - 1068, (2005).

28. Y., Wan, Chen, Q., and Zhang, B. M. Image enhancement based on equal area dualistic subimage histogram equalization method. IEEE Trans. Consum. Electron.,45,68-75, (1999).

29. K. Dimililer, A. Hesri and Y. Kirsal Ever, Lung Lesion Segmentation using Gaussian Filter and Discrete Wavelet Transform, The Journal of ITM Web of Conferences, EDP Sciences, ISSN 2271-2097, 11, (2017).

30. I. J. Bush and K. Dimililer, Static and Dynamic Pedestrian Detection Algorithm for Visual Based Driver Assistive System, The Journal of ITM Web of Conferences, EDP Sciences, ISSN 2271-2097, 9, (2017).
31. K. Dimililer, A. Hesri and Y. Kirsal Ever, Lung Lesion Segmentation using Gaussian Filter and Discrete Wavelet Transform, The 2017 International Conference on Information Science and Technology, 11, (2017).

32. I. J. Bush and K. Dimililer, Static and Dynamic Pedestrian Detection Algorithm for Visual Based Driver Assistive System, The 2016 International Conference Applied Maths, Computational Science and Systems Engineering (AMCSE2016), 9, (2017). 